\title{
Four methods of data regularization
}

Sergey Fomel and Yang Liu

(The University of Texas at Austin)

\section{SUMMARY}

When seismic data regularization is formulated as an inverse problem, it requires mathematical regularization, a method for imposing constraints on the reconstructed data. Mathematical regularization can take four different forms: a differential operator (such as a prediction-error filter or a plane-wave destructor), an integral operator (such as a recursive inverse of prediction-error filtering or

a plane-wave constructor), a sparseness constraint in a special domain (such as Fourier or seislet), or a shaping operator. Similar results can be achieved with different methods but at a different computational cost. Using both onedimensional toy examples and seismic field data applications, we compare and illustrate properties of the four methods of data regularization. 